\title{
Polyacetylenes in Tissue Cultures of Campanula glomerata
}

\author{
Norie TANAKA*, Yoshikazu Yamada*, Koichiro ShImomura** \\ and Kanji IsHIMARU*
}

Campanula glomerata L. var. dahurica Fish. is distributed in the temperate regions in East Siberia, Korea and Japan(rarely found on the grassy plain around Mt. Aso, Kyushu island). Although the plant is very important as a horticultural plant(as garden and pot flowers), there have not been sufficient studies on the secondary metabolites. Recently, Tada et al. reported the existence of polyacetylene constituents in the intact plant ${ }^{1)}$. In the course of chemical and tissue culture studies on campanulaceous plants, some tissue cultures (in vitro shoot, callus and hairy root cultures) of this plant were established and the production of polyacetylenes in these cultures was determined.

Seeds of C. glomerata L. var. dahurica Fish., purchased from a market, were sterilized by the usual method ( $2 \% \mathrm{NaOCl}, 10 \mathrm{~min}$.) and germinated aseptically on $0.5 \%$ agar medium containing $0.5 \%$ sucrose. The axenic plants were transferred to hormone-free Murashige-Skoog $(\mathrm{MS})^{2)}$ solid medium (solidified with $2.5 \mathrm{~g} / \mathrm{l}$ gelrite) and subcultured under light (16 hr photoperiod per a day, 3000 $1 \mathrm{x})$.

The leaf segments $(3 \mathrm{~mm} \times 3 \mathrm{~mm})$ of in vitro plantlets were cut off and placed on MS solid medium supplemented with $2 \mathrm{mg} / l$ naphthaleneacetic acid and $0.1 \mathrm{mg} / l$ benzyladenine. After about 2 months of culture in the dark, callus tissues, derived on the segments, were cut off, transferred to the same medium and subcultured in the dark.

The leaf and stem segments of in vitro plants were infected with Agrobacterium rhizogenes MAFF 03-01724 by co-culture method (4 days of co-culture time) as mentioned before ${ }^{1}$. The hairy roots, appeared from the infected segments, were cut off, eliminated the bacteria and were subcultured(at 6 week intervals) on hormone-free MS solid medium in the dark. One clone which showed the best growth was selected and used for this experiment. The transformation of the hairy roots was proved by the detection of opine, mikimopine ${ }^{3)}$ using paper electrophoresis.

Fresh hairy roots $\left(c a .50 \mathrm{mg}\right.$ ) were inoculated into hormone-free Gamborg B5(B5) ${ }^{4}$ and Woody Plant $(\mathrm{WP})^{5)}$ liquid media $(50 \mathrm{~m} l$ in $100 \mathrm{~m} l$ flask) and cultured $(100 \mathrm{rpm}$, on a rotary shaker) in the dark. The growth (root weight) and polyacetylene production were determined periodically (once a week for 8 weeks).

Sample preparation and HPLC conditions for the determination of polyacetylenes were the same as mentioned before ${ }^{6}$.

All culture media, MS (containing $30 \mathrm{~g} / l$ sucrose), B5 (containing $20 \mathrm{~g} / l$ sucrose) and WP(containing $20 \mathrm{~g} / l$ sucrose) were adjusted to $\mathrm{pH} 5.7$ before autoclaving at $121^{\circ} \mathrm{C}$ for $15 \mathrm{~min}$. All cultures were placed at $25^{\circ} \mathrm{C}$. Data shown here are the mean of three experiments.

The growth [dry weight $(\mathrm{dw})]$ of the hairy roots cultured in hormone-free B5 and WP liquid media

* Department of Applied Biological Sciences, Faculty of Agriculture, Saga University, 1 Honjo, Saga 840, Japan

** Tsukuba Medicinal Plant Research Station, National Institute of Health Sciences, 1 Hachimandai, Tsukuba, Ibaraki 305, Japan 


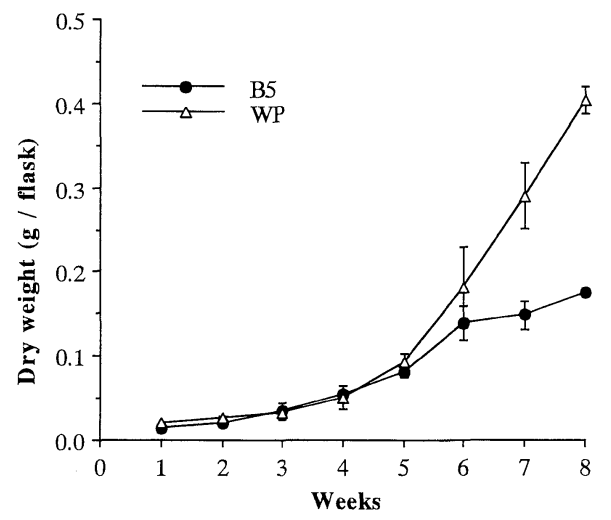

Fig. 1 Growth of Campanula glomerata hairy roots cultured in B5 and WP liquid media. Bars represent standard errors.

is shown in Fig. 1. In both media, the hairy roots continuously proliferated throughout the culture period. Particularly, in WP medium, the amount of the roots rapidly increased at the latter stage (after 5 weeks) of the culture whose maximum level ( $0.40 \mathrm{~g}$ as $\mathrm{dw}$ per a flask, at week 8) was almost double compared to that observed in B5 medium.

Recently, three polyacetylene compounds(lobetyol, lobetyolin and lobetyolinin, Fig. 2)were clarified to be produced in some hairy roots of campanulaceous plants ${ }^{1,6,7)}$. These compounds are expected to be useful chemotaxonomic markers of Campanulaceae. The content of these polyacetylenes in C. glomerata L. var. dahurica Fish. hairy roots cultured in B5 and WP liquid media was also determined(Fig. 3). In both media, a high content of lobetyolin(monoglucoside of<smiles>CC=CC#CC#CC(O)C(O)C=CCCO</smiles>

lobetyol : $\mathbf{R}=\mathrm{H}$

lobetyolin : $\mathbf{R}=\mathbf{G l c}$ lobetyolinin : $R=$ Glc $^{6}$-Glc

Fig. 2 Structures of polyacetylenes.
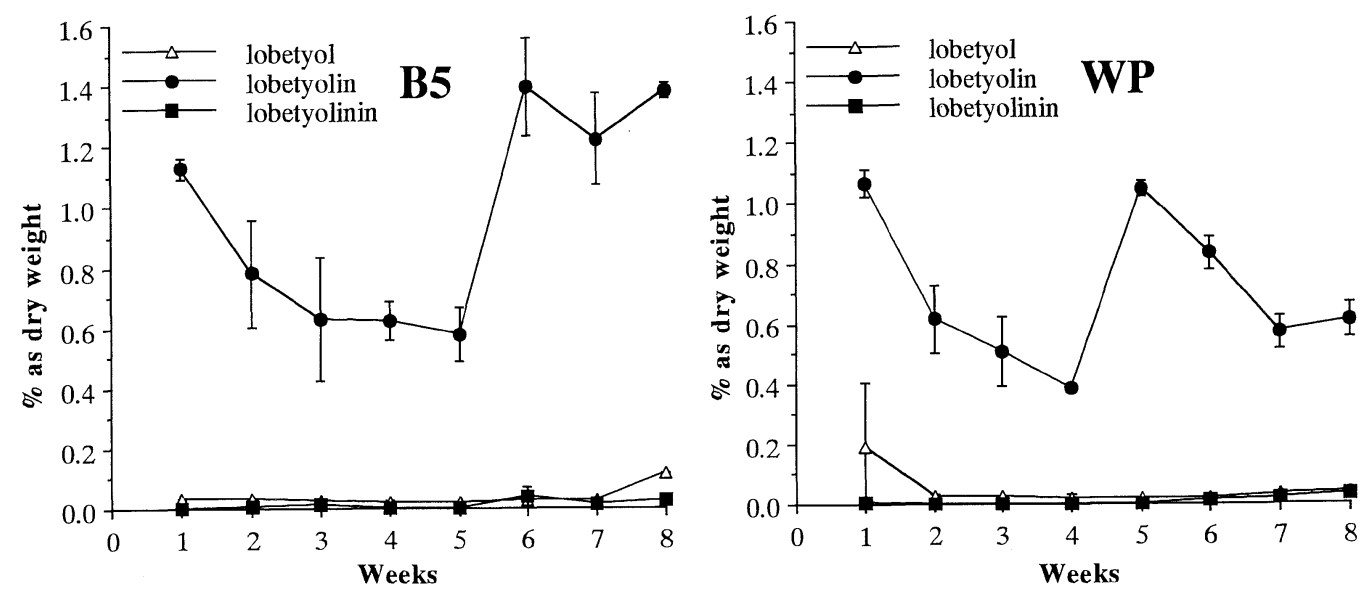

Fig. 3 Polyacetylene content of Campanula glomerata hairy roots cultured in B5 and WP liquid media.

Bars represent standard errors. 
lobetyol) was observed throughout the culture period $(0.58 \sim 1.40 \%$ in B5 and $0.39 \sim 1.06 \%$ as dw in $\mathrm{WP}$ ). At the later period (after week 5) of the culture, lobetyolin content in B5 exceeded that in WP which was in contrast with the case of the growth(Fig. 1) observed at the same period. The contents of lobetyol and lobetyolinin(diglucoside of lobetyol) were fairy poor in these media.

Polyacetylene contents in the other tissue cultures (callus and in vitro shoot) and the intact plant ${ }^{1)}$ (leaf portion) were much lower than those of the hairy roots; $i$. e., contents of lobetyolin in callus, shoot culture and leaf of intact plant were $0.01 \%, 0.07 \%$ and $0.20 \%$ as dw, respectively (lobetyol and lobetyolinin were not detected in these tissues).

By the first chemical research on the secondary metabolites in some tissue cultures (hairy root, callus and shoot cultures) of C. glomerata L. var. dahurica Fish., it was demonstrated that the hairy roots characteristically yield a much larger amount of polyacetylenes(especially the glucoside, lobetyolin) compared to those produced in the other tissues of the plant.

\section{Acknowledgements}

This work was supported in part by the subsidy from The San-Ei Gen Foundation for Food Chemical Research and by the Ministry of Health and Welfare, Science Research Fund Subsidy granted to the Japan Health Science Foundation.

(Accepted January 22, 1996)

\section{References}

1) Tada, H., K. Shimomura, K. Ishimaru. 1995. J. of Plant Physiology 145: 7-10.

2) Murashige, T., F. Skoog. 1962. Physiol. Plant., 15: 473-497.

3) Isogai, A., N. Fukuchi, M. Hayashi, H. Kamada, H. Harada, A. Suzuki. 1990. Phytochemistry, 29: 31313134.

4) Gamborg, O. L., R. A. Miller, K. Ojima. 1968. Exp. Cell Res., 50: 151-158.

5) Lloyd, G. B., B. H. McCown. 1980. Int. Plant. Prop. Soc., 30: 421-427.

6) Ishimaru, K., H. Arakawa, S. Sadoshima, Y. Yamaguchi, 1993. Plant Tissue Culture Letters, 10: 191-193.

7) Ishimaru, K., H. Arakawa, M. Yamanaka, K. Shimomura, 1994. Phytochemistry, 35: 365-369. 